Pensamiento Crítico Vol. $20 \mathrm{~N}^{\circ}$ 1, pp. 25-41

\title{
Estimación de modelos de volatilidad en series de rendimientos bursátiles: 2000-2014
}

Estimating volatility models in yields of stock market: 2000-2014

[Recepción: Marzo de 2015 / Conformidad: Mayo 2015]

Rafael Bustamante Romani*

\section{RESUMEN}

Las series temporales de alta frecuencia observadas en los mercados financieros y cambiarios se caracterizan por ser asimétricas, leptocúrticas, agrupamiento de la volatilidad, mostrar una elevada persistencia en volatilidad, correlaciones en los cuadrados, efecto leverage, etc. Estas características son las que se conoce en la literatura econométrica como hechos estilizados. Para recoger estas características de las series temporales se han planteado modelos no lineales, entre los que se pueden destacar los modelos $\mathrm{ARCH}$ y $\mathrm{GARCH}$ y todas sus posibles variantes. En este trabajo, se analiza los distintos resultados obtenidos de la estimación de los modelos propuestos, aplicados a series de rendimientos de índices bursátiles.

* Docente investigador de la UNMSM. Cursos de enseñanza: Econometría Financiera, Microeconometría, Macroeconometría, Valorización de empresas, estadística para la toma de Decisiones Empresariales. MBA CENTRUM, PUCP. Con estudios de Doctorado en Economía UNAM, México. 


\title{
Pensamiento Crítico Vol. 20. Nº
}

Existen diferentes métodos para la medición del agrupamiento de la volatilidad en las series financieras, en las cuales el supuesto sobre la distribución del error determina la estructura de la función de log verosimilitud estimada. En este documento se explota la flexibilidad de los modelos $\mathrm{ARCH}$ para capturar los agrupamientos de la volatilidad de los rendimientos. Los resultados indican que el modelo GARCH $(3 / 7,3 / 7)$ en la varianza supera otro tipo de especificación, que trate de medir el agrupamiento de la volatilidad de la principales índices bursátiles del mundo.

Palabras clave: rentabilidad, series de tiempo, $\mathrm{ARCH}, \mathrm{GARCH}$

Clasificación JEL: G14

\begin{abstract}
The time series of high frequency observed in the financial and currency markets are characterized by asymmetric, leptokurtic, volatility clustering, show a high persistence in volatility, correlations in the Square, leverage effect, etc. These features are known in the econometric literature as stylized facts. To collect these characteristics of the time series have been raised nonlinear models, among which stand out the ARCH and GARCH models and their possible variants each. In this paper, we will - analyze the different results obtained from the estimation of the proposed models, applied to yields of stock indices.

There are different methods for measuring volatility clustering in financial series, in which the assumption of the error distribution determines the structure of the estimated log likelihood function. In this document the flexibility of ARCH models is exploited to capture the groupings of the volatility of returns. The results indicate that the GARCH ( 3/7, 3/7) models in variance exceeds other specification , try to measure the volatility clustering of the major stock indexes in the world.
\end{abstract}

Keywords: rate of return, time series, $\mathrm{ARCH}, \mathrm{GARCH}$ 


\section{Rafael Bustamante Romaní}

\section{Introducción}

La creciente inestabilidad de las principales variables económicas y financieras, así como de los mercados financieros bursátiles desde las últimas décadas del siglo pasado creó la necesidad de una gestión activa del riesgo financiero.

Uno de los múltiples riesgos financieros que enfrentan los inversionistas son los riesgos de mercado; entendido como la pérdida potencial que puede sufrir debido a las variaciones en los precios que se registran en los mercados financieros; o en fluctuaciones de los factores de riesgo tales como: las tasas de interés y los tipos de cambio, entre otros.

Uno de los hechos más característicos de las variables financieras y de sus rendimientos es que su volatilidad cambia con el tiempo. Además, en algunas de ellas existe acumulación de la misma volatilidad donde comportamientos de las rentabilidades. Asimismo conocer esta volatilidad resulta crucial debido a que un exceso de esta dificulta la planificación financiera, igualmente, podría significar enormes pérdidas y/o ganancias.

Un indicador fundamental para la cuantificación de riesgos de mercado es la volatilidad de los rendimientos de un activo financiero, la cual puede no ser directamente observable por lo que se hace necesario encontrar métodos que permitan estimar y predecir esta volatilidad. (Elescano Rojas \& Agüero Palacios, 2004).

En 1982 Robert Engle propuso una nueva variedad de procesos estocásticos denominados Procesos con Heterocedasticidad Condicional Autorregresiva (ARCH), cuyo objetivo era modelar y predecir la volatilidad vigente en las series financieras. Estos procesos se caracterizan porque presentan varianzas condicionales no constantes; aun cuando sus varianzas incondicionales lo son. Los modelos $\mathrm{ARCH}$ fueron naturalmente extendidos por (Bollerslev, Engle, \& Nelson, 1993); quienes propusieron los modelos con Heterocedasticidad Condicional Autorregresiva Generalizada (GARCH) y cuya función de varianzas condicionales corresponde a un proceso ARMA.

A partir de esta propuesta surgen variantes con nombres particulares tales como modelos ARCHM (Engle, Lilien y Robins), TARCH Zakoian), EGARCH (Nelson), A-GARCH, y N-GARCH, IGARCH, V-GARCH, entre otros. Todos estos modelos son conocidos como modelos X-ARCH (Elescano Rojas \& Agüero Palacios, 2004). 


\section{Pensamiento Crítico Vol. 20. NoI}

La propiedad de estos modelos está en considerar la información pasada de la variable y la de su volatilidad como factores altamente explicativos de su comportamiento presente y por extensión lógica de su futuro predecible. Algunas características que se encuentran con frecuencia en las variables financieras que justifican el uso de los modelos de Heterocedasticidad Condicional Autorregresiva como el agrupamiento de la volatilidad. Esto significa que en estas variables, grandes cambios tienden a ser seguidos por grandes cambios, de cualquier signo, mientras que pequeños cambios en las variables son seguidos por pequeños cambios de cualquier signo.

El objetivo de este trabajo es realizar la estimación de estos diferentes modelos de volatilidad y conseguir, dependiendo de la forma de la varianza condicional, el modelo más adecuado. Posteriormente estos modelos pueden ser utilizados en la obtención de predicciones de la volatilidad, medición de riesgo, valoración de derivados financieros, etc.

\section{Metodología econométrica}

En 1982, Robert Engle introduce por primera vez un modelo que apuntaba a incorporar en el análisis econométrico estándar, la heteroscedasticidad que se observaba en los datos; este modelo se conoce como modelo ARCH (AutoRegresive - Conditional - Heteroskedastic). El modelo ARCH es útil no solo porque captura la mayor parte de los hechos estilizados de las series financieras, sino que también porque tiene aplicación en diversas áreas. (Elescano Rojas \& Agüero Palacios, 2004).

\subsection{El modelo ARCH}

Un proceso $\mathrm{ARCH}$ se define de varias maneras, la más simple es en términos de la distribución de los errores provenientes de un modelo de regresión dinámico. Sea la variable $y_{t}$ una variable aleatoria generada por el siguiente proceso.

$$
y_{t}=x_{t}^{\prime} \beta+\varepsilon_{t}
$$

Donde $x_{t}$ es el vector de variables predeterminadas, de orden kx1, el cual puede incluir valores rezagados de la variable dependiente, y $\beta$ es un vector de parámetros a estimar. Supongamos entonces que podemos modelar la varianza condicional como un proceso autorregresivo, AR (q), es decir: 


\section{Rafael Bustamante Romaní}

$$
\varepsilon_{\mathrm{t}}^{2}=\alpha_{0}+\alpha_{1} \varepsilon_{\mathrm{t}-1}^{2}+\alpha_{2} \varepsilon_{\mathrm{t}-2}^{2}+\ldots+\alpha_{\mathrm{q}} \varepsilon_{\mathrm{t}-\mathrm{q}}^{2}+v_{\mathrm{t}}
$$

Donde $v_{t}$ es un ruido blanco. Tomando esperanza condicional de la ecuación anterior se obtiene que:

$$
\mathrm{E}_{\mathrm{t}}\left(\varepsilon_{\mathrm{t}}^{2}=h_{t}=\alpha_{0}+\alpha_{1} \varepsilon_{\mathrm{t}-1}^{2}+\alpha_{2} \varepsilon_{\mathrm{t}-2}^{2}+\ldots+\alpha_{\mathrm{q}} \varepsilon_{\mathrm{t}-\mathrm{q}}^{2}\right)
$$

Es decir la varianza condicional es:

$$
\sigma_{t}^{2}=h_{t}=\alpha_{0}+\alpha_{1} \varepsilon_{\mathrm{t}-1}^{2}+\alpha_{2} \varepsilon_{\mathrm{t}-2}^{2}+\ldots+\alpha_{\mathrm{q}} \varepsilon_{\mathrm{t}-\mathrm{q}}^{2}
$$

Con $\alpha_{0} \geq 0, \alpha_{i} \geq 0$ para $\mathrm{i}=1,2,3, \ldots, \mathrm{q}$, para así garantizar que la varianza condicional sea positiva. Se puede notar que $\varepsilon_{t-1}=y_{t-1}-x_{t-i} \beta, \mathrm{i}=1,2,3$,... .

Que no es otra cosa que el modelo ARCH (q) (Engle, 1982). Este tipo de modelos son de especial importancia cuando se quiere estimar y predecir las fluctuaciones de variables de interés, como podrían ser los precios de las acciones y otra información financiera. Asimismo, el mejorar la estimación de la varianza hace posible la determinación de intervalos de confianza de mayor precisión y eleva la calidad de la estimación de los parámetros del modelo original. Finalmente, la varianza estimada puede ser utilizada como variable explicativa en algún modelo econométrico en el que se quiera relacionar el comportamiento de una variable con la volatilidad de otra. Cabe mencionar que la especificación lineal no es la más conveniente ya que el modelo para $y_{t}$ y su varianza condicional son estimados mejor simultáneamente usando el método de estimación de máxima verosimilitud. Por ello, Engle propone trabajar con una especificación multiplicativa, cuyo ejemplo más simple es (Beltran Barco, 2002):

$$
\varepsilon_{t}=v_{t} \sqrt{\alpha_{0}+\alpha_{1} \varepsilon_{t-1}^{2}}
$$

Donde $v_{t}$ es un ruido blanco con media 0 y varianza 1 , st- 1 es independiente y $\boldsymbol{\alpha} 0$ y $\boldsymbol{\alpha} 1$ son constantes tal que $\boldsymbol{\alpha} 0>0$ y $0<\boldsymbol{\alpha} 1<1$.

Es un modelo ARCH la estructura del error y los parámetros de autocorrelación del proceso $\left\{y_{t}\right\}$ interactúan mutuamente. Cualquier shock inusualmente grande (expresado 


\section{Pensamiento Crítico Vol. 20. NoI}

en su desviación estándar) en $v_{t}$ sería asociado a una varianza persistentemente grandeen las secuencias de errores presente y futuros $\left\{\varepsilon_{t}\right\}$, siendo el valor de $\alpha_{1}$ lo que determinará la persistencia.

Es posible generalizar la especificación del proceso a través de un $\mathrm{ARCH}$ de orden mayor en el caso en que todos los shocks desde, $\mathcal{E}_{t-1}$ hasta $\varepsilon_{t-q}$ tienen un efecto directo sobre $\varepsilon_{t}$, de tal manera que la varianza condicional sigue un proceso autorregresivo de orden q.

$$
\stackrel{a}{t}_{t}=v_{t} \sqrt{a_{o}+\sum_{i=1}^{q} a_{i} a_{t-i}^{2}}
$$

Que e da origen a un modelo $\mathrm{ARCH}$ de orden q.

\subsection{El modelo GARCH}

$\mathrm{GARCH}$ es la abreviatura de Generalized Autorregresive Conditional Heteroscedasticity y da nombre a la ampliación del modelo ARCH ya comentado que realizó Bollerslev (1986) para los órdenes p,q, y Taylor (1986), para el caso específico de los órdenes 1,1. Bollersev (1986) propone una extensión a la función de varianza condicional que recibe el nombre de ARCH generalizado o GARCH. De gran utilidad en los trabajos empíricos. Bollersev sugiere la siguiente especificación para la varianza condicional (Arce, 1998). Basado en una extensión del trabajo de Engle, desarrolló una técnica que permite que la varianza condicional siga un proceso ARMA.

$$
\varepsilon_{t}=v_{t} \sqrt{h_{t}}
$$

Donde:

$$
\sigma_{v}^{2}=1 \wedge h_{t}=\alpha_{o}+\sum_{i=1}^{q} \alpha_{i} \varepsilon_{t-i}^{2}+\sum_{i=1}^{p} \beta_{i} h_{t-i}
$$

En el que se incorpora una estructura dinámica más compleja en la ecuación de la varianza, con componentes autorregresivos y de medias móviles. Se puede observar que en este caso que: 


$$
E\left(\varepsilon_{t}^{2} / \varepsilon_{t-1}, \varepsilon_{t-2}, \ldots\right)=h_{t}=\alpha_{o}+\sum_{i=1}^{q} \alpha_{i} \varepsilon_{t-i}^{2}+\sum_{i=1}^{p} \beta_{i} h_{t-i}
$$

Donde los $\boldsymbol{\alpha}$ 's y $\boldsymbol{\beta}$ 's son menores que 1, para que el modelo no sea explosivo. Así, como se ve en (10), la varianza condicional depende de una constante, de la volatilidad pasada y de la estimación de la varianza condicional en el pasado.

El punto importante es que la varianza condicional de $\varepsilon_{t}$ esta dado por $E_{t-1} \varepsilon_{t}^{2}=h_{t}$ , por lo tanto, los componentes de $h_{t}$ son los que determinan la varianza condicional $\mathrm{de} \mathcal{E}_{t}$.

La secuencia $\left\{v_{t}\right\}$ es un ruido independiente de los valores pasados de $\varepsilon_{t}$ por lo que la media condicional e incondicional de $\varepsilon_{t}$ será siempre cero.Hay que considerar que un modelo $\mathrm{ARCH}$ de orden alto puede tener una representación $\mathrm{GARCH}$ más parsimonioso, que es más sencilla de identificar y estimar.

\section{Análisis de los datos}

A continuación se procede a estimar la volatilidad de las principales bolsas de valores del mundo, con el fin de observar cual es el componente GARCH común que los caracteriza.Se trabaja con datos de frecuencia diaria para los años entre 1990 hasta finales del 2014.

\section{1 Índice de Bovespa}

Es un índice bursátil compuesto de unas 50 compañías que cotizan en la Bolsa de Sao Paulo (Bovespa:Bolsa de Valores do Estado de São Paulo, Brasil).Está compuesto por los títulos de las empresas que suponen el $80 \%$ del volumen negociado en los últimos 12 meses y que fueron negociados por lo menos el $80 \%$ de los días de cotización. Es revisado trimestralmente, para mantener el grado de representación de todas las acciones negociadas en el mercado. 


\section{Pensamiento Crítico Vol. 20. NoI}

Gráfico 1: Comportamiento del Índice Bursátil BOVESPA

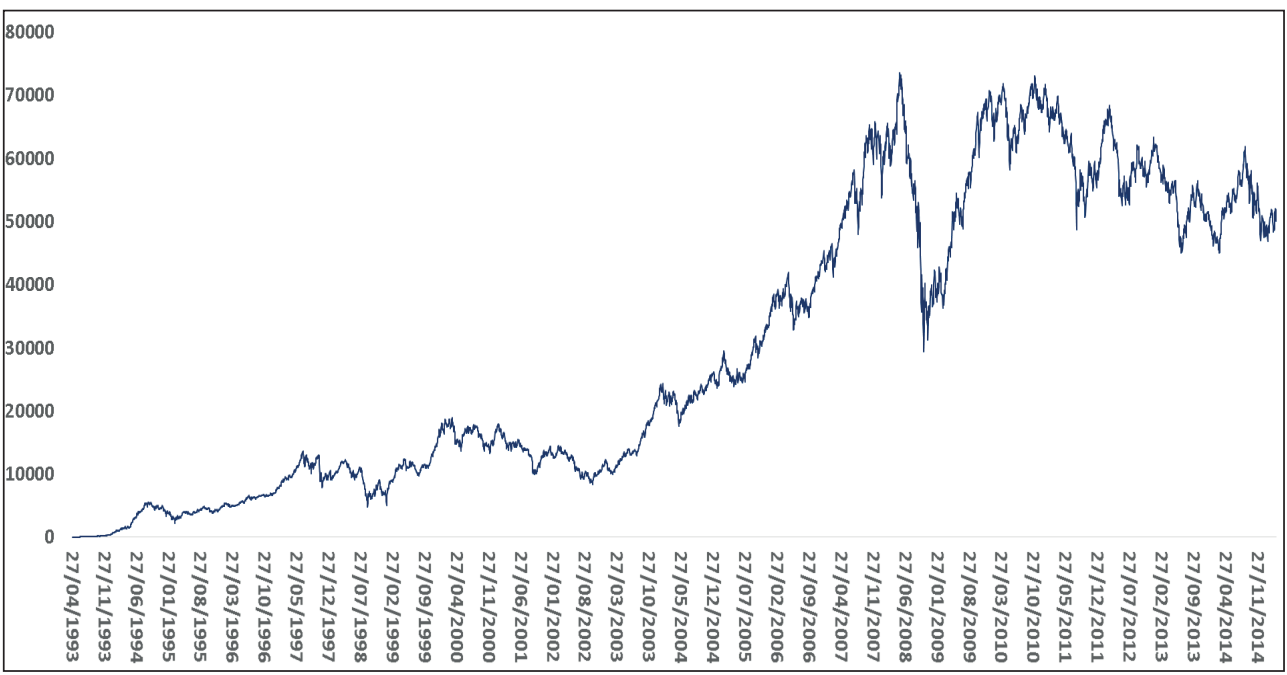

Fuente: Yahoo Finance. Elaboración propia

\section{Prueba de raíces unitarias}

Realizamos la prueba de raíces unitarias, para ello realizamos la prueba de DFGLS. Esta prueba realiza la prueba t de Dickey-Fuller modificada, propuesta por Elliott, Rothenberg y Stock (1996). La esencia de la prueba es hacer una secuencia similar a la prueba de Dickey-Fuller, excepto que la serie de tiempo es transformado a través de una regresión por mínimos cuadrados generalizados (GLS) antes de realizar la prueba. Elliott, Rothenberg y Stock y estudios posteriores han demostrado que esta prueba tiene significativamente mayor poder que las versiones anteriores de la prueba Dickey-Fuller aumentada1.Según este test vemos la rentabilidad del índice BOVESPA rechazala hipótesis nula de existencia de raíz unitaria dado que el estadístico DF-GLS es 7.5715, mayoral t- Statistic al 5\% de nivel de significancia (1.9408). A continuación presentamos el comportamiento de las rentabilidades en el Gráfico 2.

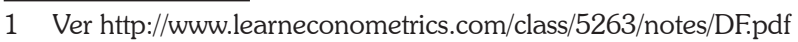




\section{Rafael Bustamante Romaní}

Gráfico 2: Comportamiento de las Rentabilidades del IBOVESPA

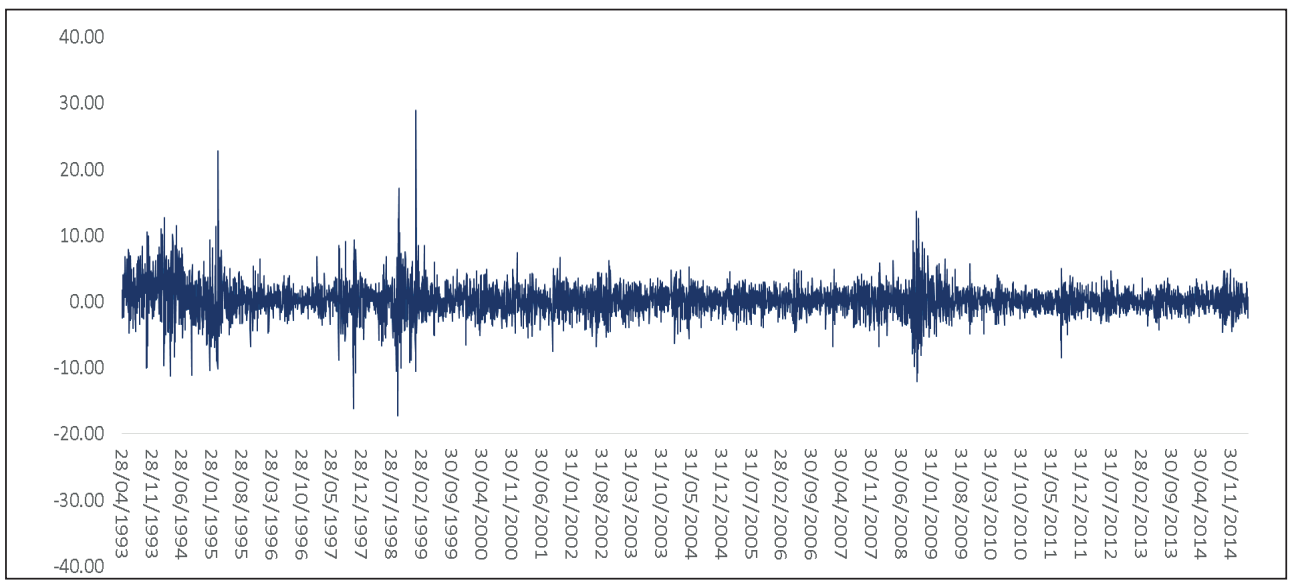

Fuente: Yahoo Finance. Elaboración propia

En en el Gráfico 2 puede observar el comportamiento de la rentabilidad es de carácter heterocedástico. Sometemos al test de Jarquer Bera rechazándose la hipótesis de normalidad. A partir de las funciones de correlación simple y parcial de los residuos del modelo estimado, vemos que la forma de las gráficas sugieren un $\mathrm{GARCH}$ (p. 4) donde el componente p será buscado con los criterios de información. El criterio Akaike (AIC) es una medida de la calidad relativa de un modelo econométrico que proporciona un medio para la selección del modelo. Maneja un trade-off entre la bondad de ajuste del modelo y la complejidad del modelo. Ofrece una estimación relativa de la información pérdida cuando se utiliza un modelo determinado para representar el proceso que genera los datos. El criterio de información bayesiano (BIC) o el más general criterio de Schwarz (SBC también, SBIC) sirve para la selección de modelos entre un conjunto finito de modelos y está estrechamente relacionado con el Criterio de Información de Akaike (AIC).Cuando el ajuste de modelos, es posible aumentar la probabilidad mediante la adición de parámetros, pero si lo hace puede resultar en sobreajuste. Tanto el BIC y AIC resuelven este problema mediante la introducción de un término de penalización para el número de parámetros en el modelo, el término de penalización es mayor en el BIC que en el AIC2.

2 El BIC fue desarrollado por Gideon E. Schwarz, quien dio un argumento bayesiano a favor de su adopción. 1 Akaike también desarrolló su propio formalismo Bayesiano, que ahora se conoce como la ABIC por Criterio de Información Bayesiano de Akaike. 


\section{Pensamiento Crítico Vol. 20. NoI}

Dichos criterios nos han llevado a elegir un $\operatorname{GARCH}(3,3)$ que es el que presentó menor AKAIKE. Como se puede observar en la Figura $\mathrm{N}^{\circ}$ hemos elegido un GARCH $(3,3)$ según los criterios de información AIC y BIC.

Cuadro 1: Estimación del Modelo GARCH $(3,3)$

\begin{tabular}{|c|c|c|c|c|c|c|}
\hline \multirow{2}{*}{\multicolumn{4}{|c|}{ Distribution: Gaussian }} & \multicolumn{2}{|c|}{ Number of obs $=$} & 5443 \\
\hline & & & & Wald chi2(.) & $=$ & 0.001 \\
\hline \multicolumn{4}{|c|}{ Log likelihood = -11519.38 } & Prob > chi2 & $=$ & 0 \\
\hline RIBOVESPA & Coef. & Std. Err. & $\mathbf{z}$ & $\mathbf{P}>\mathbf{z}$ & \multicolumn{2}{|c|}{ [95\% Con Interval] } \\
\hline CONSTANTE & 0.124 & 0.026 & 4.790 & 0.000 & 0.074 & 0.175 \\
\hline ARCH(1) & 0.143 & 0.009 & 16.540 & 0.000 & 0.126 & 0.160 \\
\hline ARCH(2) & 0.040 & 0.015 & 2.730 & 0.006 & 0.011 & 0.069 \\
\hline ARCH(3) & 0.049 & 0.013 & 3.730 & 0.000 & 0.023 & 0.074 \\
\hline GARCH(1) & 0.133 & 0.027 & 5.010 & 0.000 & 0.081 & 0.185 \\
\hline GARCH(2) & 0.673 & 0.031 & 21.450 & 0.000 & 0.611 & 0.734 \\
\hline GARCH(3) & 0.684 & 0.022 & 30.770 & 0.000 & 0.640 & 0.727 \\
\hline CONSTANTEGARCH & -4.001 & 0.105 & -37.990 & 0.000 & -4.208 & -3.795 \\
\hline
\end{tabular}

Fuente: Yahoo Finance. Estimaciones econométricas del autor

Se observa todos los coeficientes son significativos. A partir de este modelo se realiza la predicción de la varianza condicional de la rentabilidad del BOVESPA, el cual demuestra ser consistente con los eventos de volatilidad del comportamiento de la serie.

Gráfico 3: Estimación del Modelo GARCH $(3,3)$
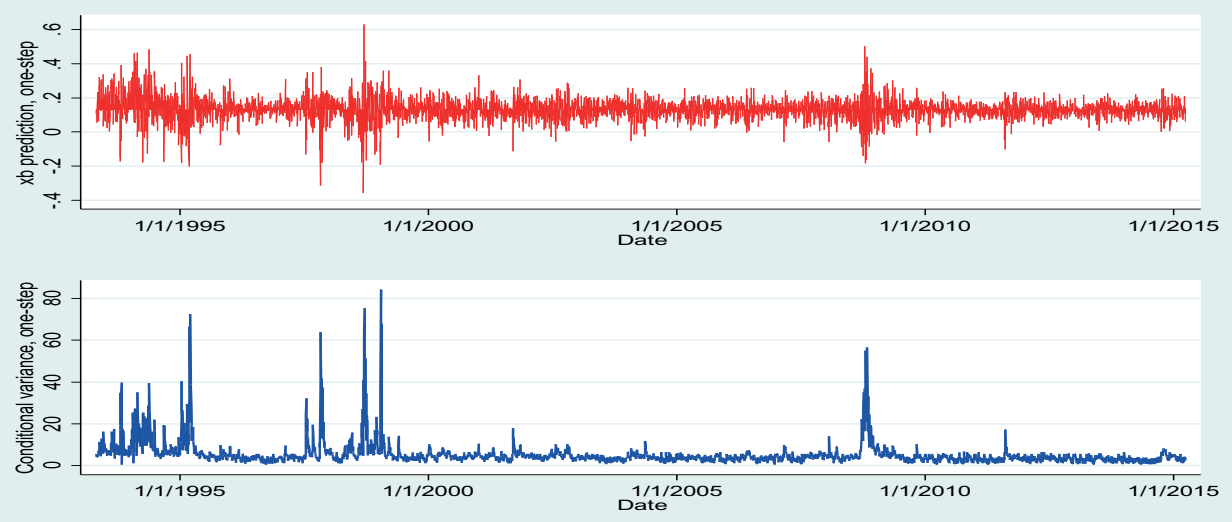

Fuente: Yahoo Finance. Estimaciones econométricas del autor 


\section{Rafael Bustamante Romaní}

\section{2. Índice de S\&P500}

El índice se basa en la capitalización bursátil de 500 grandes empresas que poseen acciones que cotizan en las bolsas NYSE o NASDAQ. Los componentes del índice y su ponderación son determinados por S\&P Dow Jones Indices. Se diferencía de otros índices de mercados financieros de Estados Unidos, en la diversidad de los rubros que lo conforman y en su metodología de ponderación.

Gráfico 4: Comportamiento del Índice Bursátil SP500

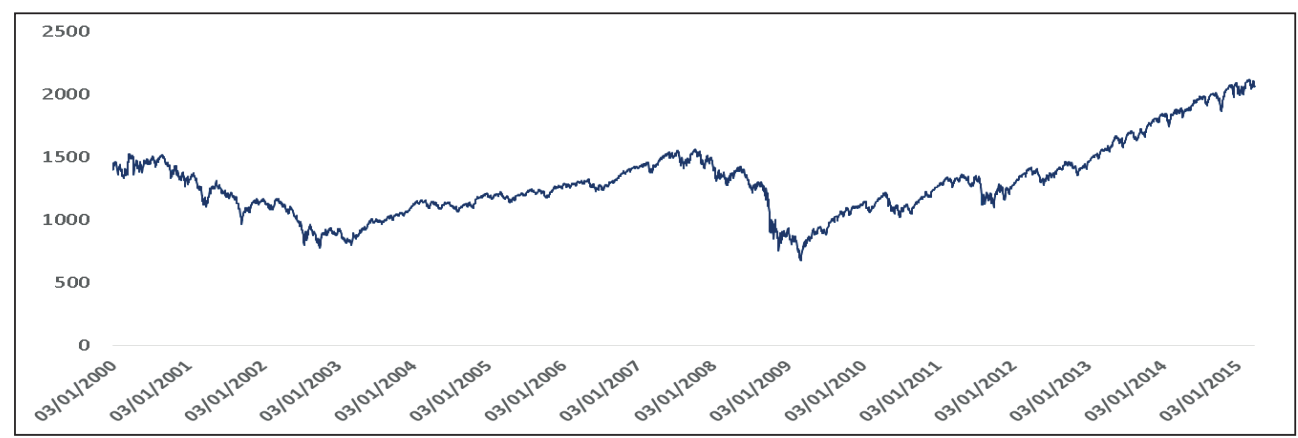

Fuente: Yahoo Finance. Elaboración propia

A continuación realizamos la prueba de DFGLS para las rentabilidades del índice. Según este test se rechaza la hipótesis nula de existencia de raíz unitaria dado que el estadístico DF-GLS es 2.565, mayor al t Statistic al 5\% de nivel de significancia (1.941). Luego realizamos la gráfica de la rentabilidad del índice SP500.

Gráfico 5: Comportamiento de las Rentabilidades del SP500

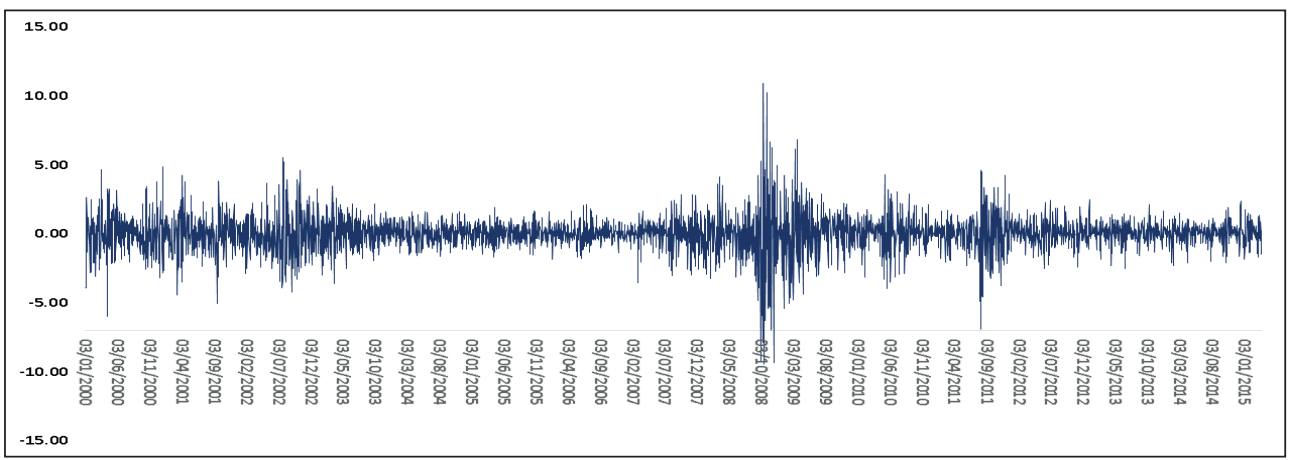




\section{Pensamiento Crítico Vol. 20. NoI}

Se observa el comportamiento heterocedástico de esta rentabilidad. Sometemos al test de Jarquer Bera rechazándose la hipótesis de normalidad. A partir de las funciones de autocorrelogramas encontramos que el modelo adecuado para explicar la evolución de la rentabilidad de índice S\&P5OO es un ARMA $(3,3)$.

Cuadro 2: Estimación del Modelo GARCH (3,4)del SP500

\begin{tabular}{|c|c|c|c|c|c|c|}
\hline \multirow{2}{*}{\multicolumn{4}{|c|}{ Distribution: Gaussian }} & \multicolumn{2}{|c|}{ Number of $\mathrm{O}=$} & 3832 \\
\hline & & & & Wald chi2(3) & $=$ & 28.97 \\
\hline Log likelihooc & $d=-5616.9$ & & & Prob > chi 2 & $=$ & o \\
\hline RSP500 & Coef. & Std. Err. & $\mathbf{Z}$ & $P>Z$ & [95\% Conf. & Interval] \\
\hline CONSTANTE & 0.039 & 0.015 & 2.650 & 0.008 & 0.010 & 0.067 \\
\hline $\operatorname{AR}(3)$ & 0.462 & 0.257 & 1.800 & 0.072 & -0.041 & 0.964 \\
\hline MA(1) & -0.076 & 0.021 & -3.540 & 0.000 & -0.118 & -0.034 \\
\hline MA(3) & -0.493 & 0.251 & -1.960 & 0.050 & -0.985 & 0.000 \\
\hline $\mathrm{ARCH}(1)$ & 0.102 & 0.013 & 7.690 & 0.000 & 0.076 & 0.128 \\
\hline $\mathrm{ARCH}(2)$ & 0.153 & 0.018 & 8.580 & 0.000 & 0.118 & 0.188 \\
\hline $\mathrm{ARCH}(3)$ & 0.067 & 0.015 & 4.400 & 0.000 & 0.037 & 0.097 \\
\hline GARCH(1) & -0.109 & 0.027 & -4.030 & 0.000 & -0.162 & -0.056 \\
\hline GARCH(2) & 0.345 & 0.053 & 6.560 & 0.000 & 0.242 & 0.448 \\
\hline GARCH(3) & 0.526 & 0.022 & 23.500 & 0.000 & 0.482 & 0.569 \\
\hline GARCH(4) & 0.406 & 0.027 & 15.300 & 0.000 & 0.354 & 0.458 \\
\hline CONSTANTE] & -0.807 & 0.053 & -15.200 & 0.000 & -0.910 & -0.703 \\
\hline
\end{tabular}

Fuente: Yahoo Finance. Estimaciones econométricas del autor

Como se puede observar del modelo todos los coeficientes son significativos. Presentamos la estimación de la varianza condicional obtenida a partir del modelo.

Gráfico 6: Estimación de la rentabilidad y volatilidad condicional del modelo GARCH $(3,4)$
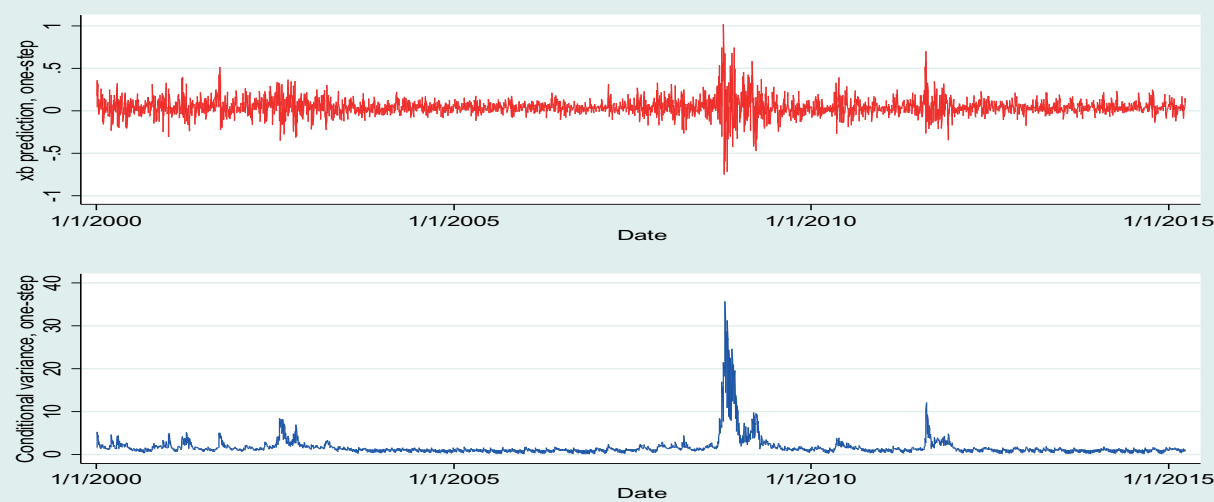

Fuente: Yahoo Finance. Estimaciones econométricas del autor 


\section{Rafael Bustamante Romaní}

En la figura nueve se puede observar que el comportamiento de la varianza condicional estimada es consistente con los periodos de volatilidad de la rentabilidad de la serie.

\section{3. Índice de IBEX 35}

El índice IBEX 35 es el principal índice bursátil de referencia de la bolsa española elaborado por Bolsas y Mercados Españoles (BME). Está formado por las 35 empresas con más liquidez que cotizan en el Sistema de Interconexión Bursátil Electrónico (SIBE) en las cuatro Bolsas Españolas (Madrid, Barcelona, Bilbao y Valencia). Es un índice ponderado por capitalización bursátil; es decir, al contrario que índices como el Dow Jones, no todas las empresas que lo forman tienen el mismo peso.

Gráfico 7: Índice de IBEX 35

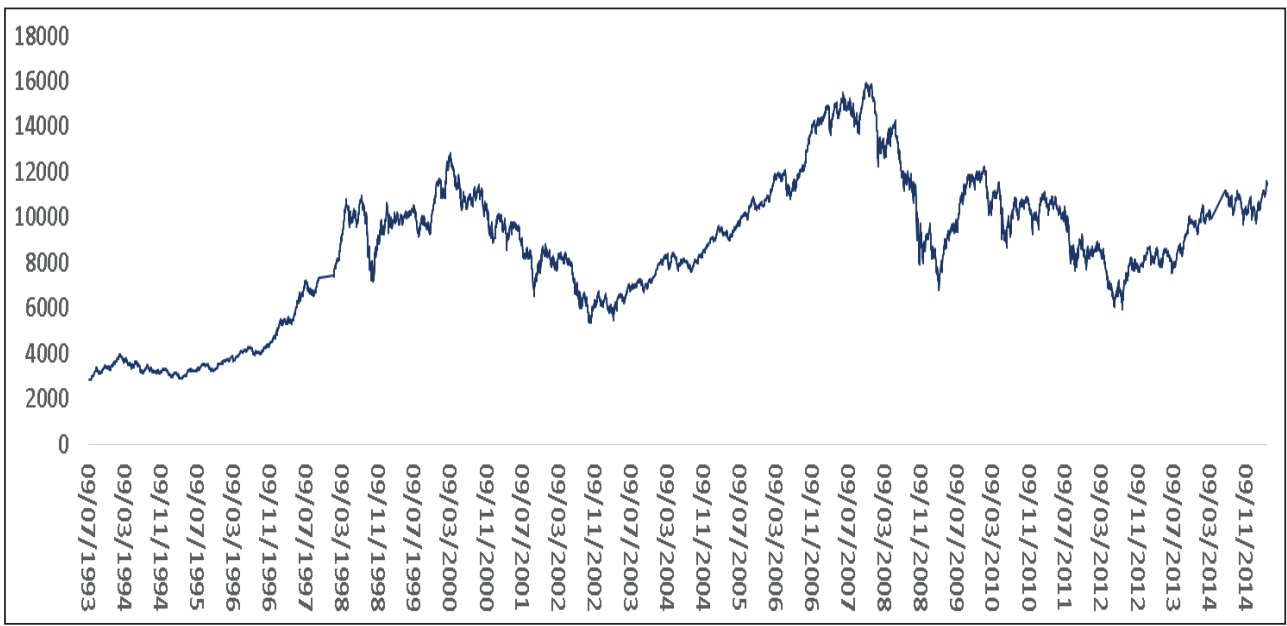

Fuente: Yahoo Finance. Elaboración propia

A continuación realizamos la prueba de raíces unitarias para las rentabilidades del índice IBEX 35, para ello realizamos la prueba de DFGLS. Según este test vemos la rentabilidad del índice rechaza la hipótesis nula de existencia de raíz unitaria dado que el estadístico DF-GLS es 70.61, mayor al t Statistic al 5\% de nivel de significancia (1.941). Realizamos una gráfica de la evolución de la rentabilidad del índice. 


\section{Pensamiento Crítico Vol. 20. NoI}

Gráfico 8: Comportamiento de las Rentabilidades del IBEX35

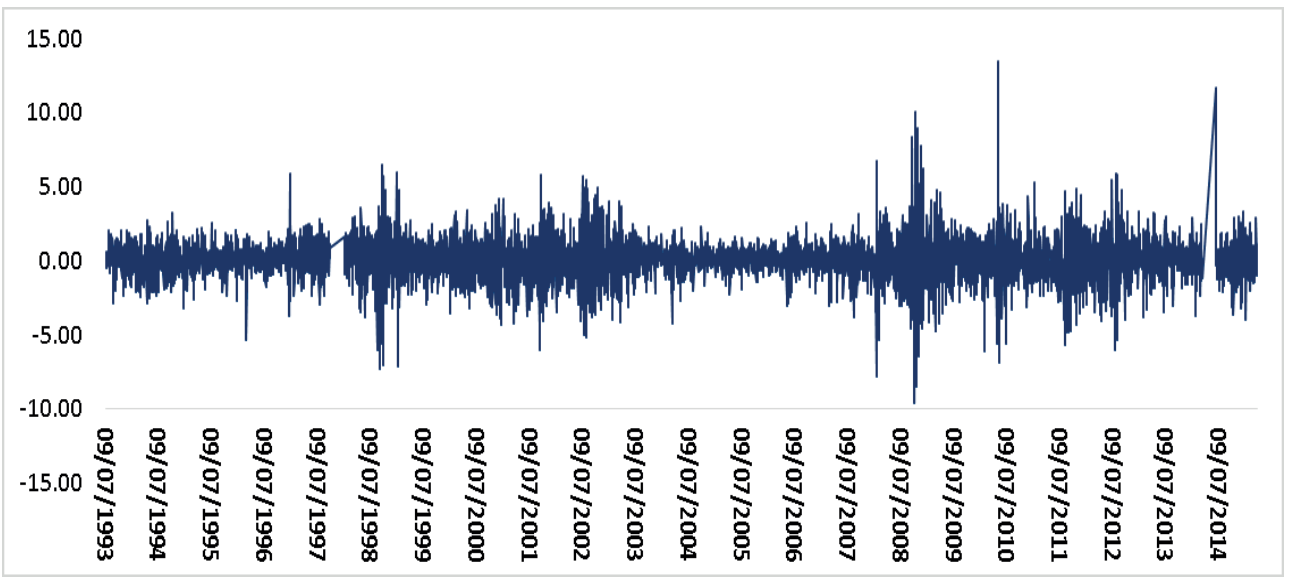

Fuente: Yahoo Finance. Elaboración propia

Sometemos al test de Jarquer Bera rechazándose la hipótesis de normalidad. A partir de las funciones de correlación simple y parcial. Se encuentra que el modelo adecuado para explicar la evolución de la rentabilidad de índice IBEX35 es un ARMA $(1,0)$.A continuación a partir de la gráfica de las funciones de correlación simple y parcial de los residuos del modelo estimado llegamos a la conclusión que la forma de las gráficas sugieren un $\mathrm{GARCH}(\mathrm{p}, 4)$ donde el componente p será obtenido con los criterios de información de Akaike (AIC) donde EL modelo elegido es un GARCH $(0,1)$.

Cuadro $\mathrm{N}^{\circ}$ 3. Estimación del Modelo GARCH $(1,1)$

\begin{tabular}{|c|c|c|c|c|c|c|}
\hline \multicolumn{4}{|c|}{ 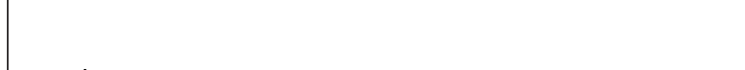 } & Number of 0 & $=$ & 5493 \\
\hline \multicolumn{4}{|c|}{ Distribution: Gaussian } & Wald chi2(1) & $=$ & 909.18 \\
\hline \multicolumn{4}{|c|}{ Log likelihood = 7327.832} & Prob >chi2 & $=$ & 0 \\
\hline RIBEX35 & Coef. & Std. Err. & $z$ & $P>z$ & [95\% Conf. & Interval] \\
\hline CONSTANTE & 0.00 & 0.00 & -2.21 & 0.03 & 0.00 & 0.00 \\
\hline $\operatorname{AR}(1)$ & -0.60 & 0.02 & -30.15 & 0.00 & -0.64 & -0.56 \\
\hline $\mathrm{ARCH}(1)$ & 0.25 & 0.00 & 56.45 & 0.00 & 0.24 & 0.26 \\
\hline CONSTANTE 2 & 0.00 & 0.00 & 506.44 & 0.00 & 0.00 & 0.00 \\
\hline
\end{tabular}




\section{Rafael Bustamante Romaní}

Como se puede observar del modelo todos los coeficientes son significativos.A continuación presentamos la estimación de la de la rentabilidad de IBEX35, así como la estimación de la varianza condicional de la misma obtenido a partir del modelo GARCH $(0,1)$.

Gráfico 9: Estimación del Modelo GARCH $(5,6)$
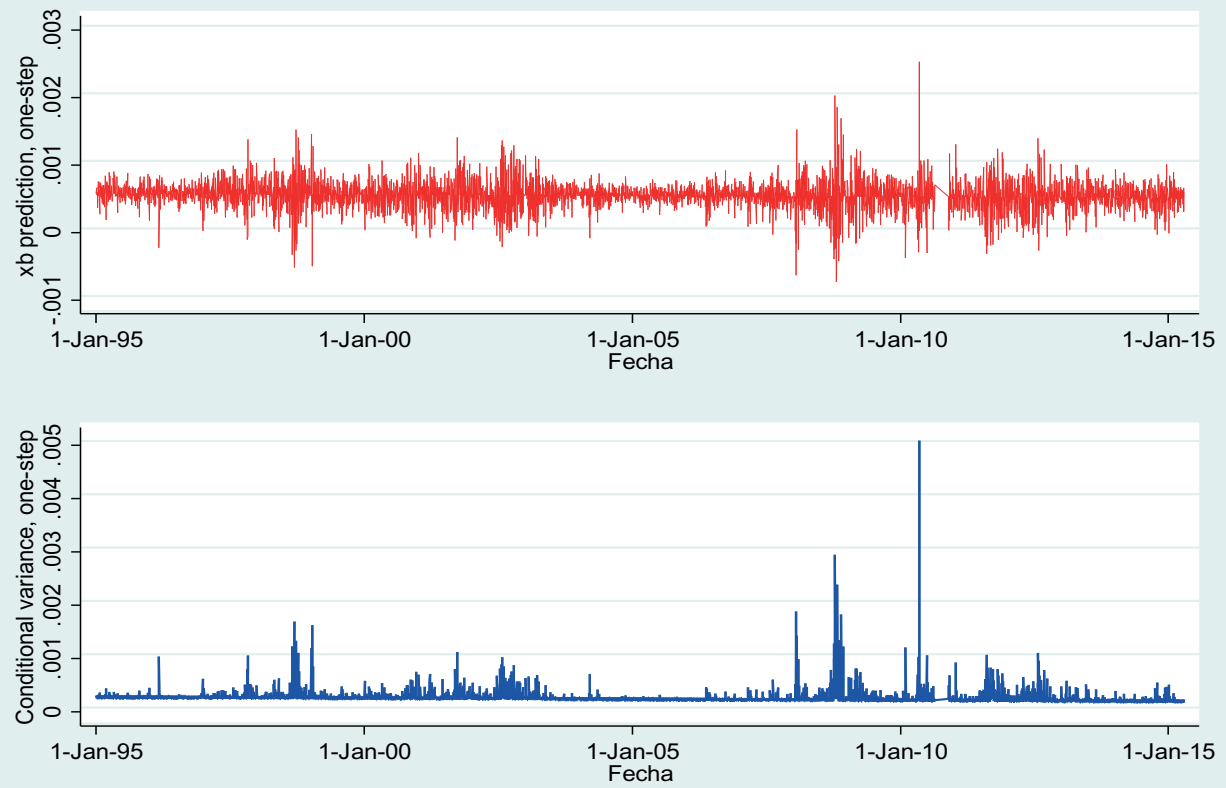

Fuente: Yahoo Finance. Estimaciones econométricas del autor

En la figura nueve se puede observar que el comportamiento de la varianza condicional estimada es consistente con los periodos de volatilidad de la rentabilidad de la serie.

Finalmente cabe mencionar que en el estudio de todas las series, se buscó la posibilidad de estimación de componentes ARCH-M y EGARCH llegando a ser no significativos dichos modelos. 


\section{Pensamiento Crítico Vol. 20. NoI}

\section{Conclusiones y recomendaciones}

La utilidad de estos modelos y su relevancia depende del agente económico interesado en usarlos, por un lado no son una herramienta útil para la medición del riesgo en el sector real, ya que no se pueden pronosticar suficientes datos para llevar a cabo una predicción que proporcione información relevante para gestionar de manera efectiva el riesgo al que el sector privado está expuesto. Sin embargo estos modelos, y estos resultados en particular, son efectivos en el sector financiero donde, para ciertas áreas (portafolios de mesas de dinero y/o de fondos de pensiones), se requiere medir el riesgo mediante la incorporación continua de información reciente; ya que el modelo GARCH arroja una tendencia acertada que permite estimar la volatilidad real observada en el periodo considerado, donde para cada índice bursátil se encontraron los siguientes modelos:

Encontramos que el modelo adecuado para explicar la evolución de la rentabilidad del índice IBOVESPA es un GARCH $(3,3)$, sin un componente autorregresivo en la rentabilidad de la series.

Se encuentra que el modelo adecuado para explicar la evolución de la rentabilidad del índice SP500 es un GARCH $(3,4)$, con un componente autorregresivo ARMA $(3,3)$ en la rentabilidad de la series.

Se encuentra que el modelo adecuado para explicar la evolución de la rentabilidad del índice IBEX35 es un GARCH $(1,1)$, con un componente autorregresivo ARMA $(1,0)$ en la rentabilidad de la series.

\section{Referencias bibliográficas}

Arce, R. (1998). Introducción a los Modelos Autorregresivos con Heterocedasticidad Condicional (ARCH). Instituto Klein. Obtenido de http://www.uam.es/otroscentros/ klein/doctras/doctra9806.pdf

Beltran Barco, A. (2002). Econometría de Series de Tiempo. No. Obtenido de http:// econometriaii.files.wordpress.com/2010/01/beltran.pdf 


\section{Rafael Bustamante Romaní}

Bollerslev, Engle, \& Nelson. (1993). ARCH Models. The Handbook ofEconometrics, 4, 1 - 90 .

Elescano Rojas, A., \& Agüero Palacios, Y. (Junio de 2004). Modelos ARCH: una aplicación en el pronóstico de la volatilidad de acciones cotizadas en la Bolsa de Valores de Lima. PESQUIMAT Revista de la Paco CC. MM., Vol. VII. (N” 1), pág. 64 - 79. Obtenido de http://revistasinvestigacion.unmsm.edu.pe/index.php/matema/ article/download/9318/8137

Bollerslev, Tim, Chou, Ray Y. \& Kroner, Kenneth F. "ARCH Modeling in Finance: A Reviewof the Theory and Empirical Evidence". Journal of Econometrics, 52: 5-59.

Bollerslev, Tim, Engle, Robert, F. \& Nelson, Daniel B. (1994). "ARCH Models". Chapter 49 in Robert F. Engle and Daniel L. McFadden (eds.). Handbook of Econometrics, 4. Amsterdam:Elsevier Science B.V.

Bollerslev, Tim \&. Wooldridge, Jeffrey, M. (1992) "Quasi-Maximum Likelihood Estimation andInference in Dynamic Models with Time Varying Covariances". Econometric Reviews, 11: 143-172.

Bollerslev, Tim. (1998) "Answering the Skeptics: Yes, Standard Volatility Models Do Provide AccurateForecasts". International Economic Review, 39: 885-905.

Campbell, J.Y., Lo, A.W. \& MacKinlay, A.C. (1997) The Econometrics of Financial Markets PrincetonUniversity Press.

Engle, R.F. (1982). "Autoregressive Conditional Heteroscedasticity with Estimates of the Varianceof UK Inflation”. Econométrica, 50: 987-1008. 